ARTICLE

\title{
Topological vacuum bubbles by anyon braiding
}

\author{
Cheolhee Han', Jinhong Park', Yuval Gefen ${ }^{2} \&$ H.-S. Sim ${ }^{1}$
}

According to a basic rule of fermionic and bosonic many-body physics, known as the linked cluster theorem, physical observables are not affected by vacuum bubbles, which represent virtual particles created from vacuum and self-annihilating without interacting with real particles. Here we show that this conventional knowledge must be revised for anyons, quasiparticles that obey fractional exchange statistics intermediate between fermions and bosons. We find that a certain class of vacuum bubbles of Abelian anyons does affect physical observables. They represent virtually excited anyons that wind around real anyonic excitations. These topological bubbles result in a temperature-dependent phase shift of Fabry-Perot interference patterns in the fractional quantum Hall regime accessible in current experiments, thus providing a tool for direct and unambiguous observation of elusive fractional statistics.

\footnotetext{
${ }^{1}$ Department of Physics, Korea Advanced Institute of Science and Technology, 291, Daehak-ro, Yuseong-gu, Daejeon 34141, Korea. ${ }^{2}$ Department of Condensed Matter Physics, Weizmann Institute of Science, Rehovot 76100, Israel. Correspondence and requests for materials should be addressed to H.-S.S. (email: hssim@kaist.ac.kr).
} 
W hen two identical particles adiabatically exchange their positions $\mathbf{r}_{i=1,2}$, their final state $\psi$ (up to dynamical phase) is related to the initial one through an exchange statistics phase $\theta^{*}$,

$$
\psi\left(\mathbf{r}_{2}, \mathbf{r}_{1}\right)=e^{i \theta^{*}} \psi\left(\mathbf{r}_{1}, \mathbf{r}_{2}\right)
$$

with $\theta^{*}=0(\pi)$ for bosons (fermions) ${ }^{1}$.

Anyons ${ }^{2-4}$ are quasiparticles in two dimensions, not belonging to the two classes of elementary particles, bosons and fermions. Abelian anyons appear in the fractional quantum Hall $(\mathrm{FQH})$ system of filling factor $v=1 /(2 n+1), n=1,2, \cdots$. They carry a fraction $e^{\star}=v e$ of the electron charge $e$ and obey fractional exchange statistics, satisfying equation (1) with $\theta^{*}= \pm \pi v$. Two anyons gain a phase $\pm 2 \pi v$ from a braiding, whereby one winds around the other; \pm depends on the winding direction. Although fractional charges have been detected ${ }^{5-8}$, experimental measurement of statistics phase $\pi v$ has been so far elusive. Existing theoretical proposals for the measurement involve quantities inaccessible in current experiments or suffer from unintended change of a proposed setup with external parameters ${ }^{9-18}$.

In many-body quantum theory ${ }^{1}$, Feynman diagrams are used to compute the expectation value of observables. This approach invokes vacuum bubble diagrams, which describe virtual particles excited from vacuum and self-annihilating without interacting with real particles. According to the linked cluster theorem ${ }^{1}$, each diagram possessing vacuum bubbles comes with, hence is exactly cancelled by, a partner diagram of the same magnitude but of the opposite sign. Consequently, vacuum bubbles do not contribute to physical observables.

In the following, we demonstrate that this common wisdom has to be revised for anyons: a certain class of vacuum bubbles of Abelian anyons does affect observables. These virtual particles, which we call topological vacuum bubbles, wind around a real anyonic excitation, gaining the braiding phase $\pm 2 \pi v$. We propose a realistic setup for detecting them and $\theta^{\star}=\pi v$.

\section{Results}

Topological vacuum bubble. We illustrate topological vacuum bubbles. In Fig. 1a, a Feynman diagram represents interference $a_{1} a_{2}^{*}$ between processes $a_{1}$ and $a_{2}$ of propagation of a real particle. In $a_{1}$, a virtual particle-hole pair is excited then self-annihilates after the virtual particle winds around the real particle, forming a vacuum bubble, while it is not excited in $a_{2}$. The winding results in a braiding phase $2 \pi v$ and an Aharonov-Bohm phase $2 \pi \Phi / \Phi_{0}^{*}$ from the magnetic flux $\Phi$ enclosed by the winding path, contributing to the interference signal as $e^{i\left(2 \pi \Phi / \Phi_{0}^{*}+2 \pi v\right)} ; \Phi_{0}^{*}=h / e^{*}$ is the anyon flux quantum ${ }^{9,19}$.

The limiting cases of bosons $(v=0)$ and fermions $(v=1)$ imply that this bubble diagram appears together with, and is cancelled by, a partner diagram in Fig. $1 \mathrm{~b}$. The partner diagram has a bubble not encircling the real particle and involves only $2 \pi \Phi / \Phi_{0}^{*}$. The two diagrams (and their complex conjugates) yield

$$
\begin{aligned}
\text { Interference signal } & \propto \operatorname{Re}\left[e^{i\left(2 \pi \Phi / \Phi_{0}^{*}+2 \pi v\right)}-e^{2 \pi i \Phi / \Phi_{0}^{*}}\right] \\
& =-\sin (\pi v) \sin \left(2 \pi \Phi / \Phi_{0}^{*}+\pi v\right) .
\end{aligned}
$$

For bosons and fermions, the two diagrams fully cancel each other with $\sin (\pi v)=0$ in agreement with the linked cluster theorem; hence, the signal disappears. By contrast, for anyons they cancel only partially, producing the non-vanishing interference in an observable, and are topological as the braiding phase is involved. a
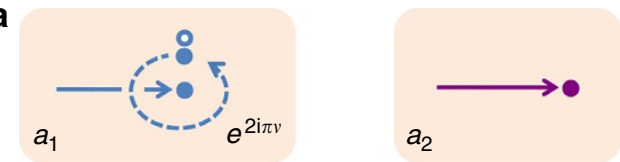

b

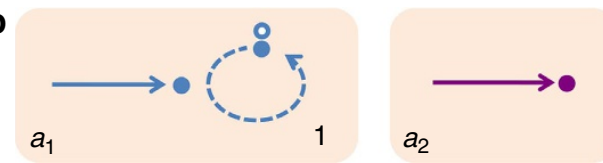

Figure 1 | Topological vacuum bubble. Feynman diagrams for interference involving a real particle and a virtual particle-hole excitation from vacuum. Full (empty) circles represent particles (holes). Solid (dashed) lines denote propagations of real (virtual) particles. (a) Diagram for the interference $a_{1} a_{2}^{*}$ of two processes: $\left(a_{1}\right.$, blue) A real particle propagates, a virtual particlehole pair is excited, then the pair self-annihilates after the virtual particle winds around the real one. $\left(a_{2}\right.$, magenta) A real particle propagates. The entire virtual process constitutes a vacuum bubble. For anyons, the bubble gains a topological braiding phase $2 \pi v$ from the winding. (b) Partner diagram of $\mathbf{a}$. Here a virtual particle, constituting another bubble, does not encircle a real one and hence gains no braiding phase. The diagrams in $\mathbf{a}$ and $\mathbf{b}$ contribute to observables for anyons, while they do not for bosons and fermions.

Interferometer setup. In Fig. 2a, we propose a minimal setup for observing topological vacuum bubbles. It is a Fabry-Perot interferometer $9,17,20-23$ in the $v=1 /(2 n+1) \mathrm{FQH}$ regime, coupled to an additional edge channel (Edge 1) via a quantum point contact (QPC1). At QPCi, there occurs tunnelling of a single anyon (rather than anyon bunching), fulfilled ${ }^{24}$ with $\gamma_{i} \ll k_{\mathrm{B}} T ; \gamma_{i}$ is the tunnelling strength and $T$ is the temperature. Gate voltage $V_{\mathrm{G}}$ is applied, to change the interferometer loop enclosing Aharonov-Bohm flux $\Phi$. The interference part $I_{D_{3}}^{\text {int }}$ of charge current at drain $D_{3}$ is measured with bias voltage $V$ applied to source $S_{1}$; the other $S_{i}$ 's and $D_{i}$ 's are grounded. Together with 'virtual' (thermal) anyon excitations in the interferometer, a voltage-biased 'real' anyon, dilutely injected at QPC1 from Edge 1 to the interferometer, forms topological vacuum bubbles, as shown below. The bubbles contribute to $I_{D_{3}}^{\text {int }}$ at the leading order $\left(I_{D_{3}}^{\text {int }} \propto \gamma_{1}^{2} \gamma_{2} \gamma_{3}\right)$ in QPC tunnelling, as Edges 2 and 3 are unbiased. It is noteworthy that in the setups previously studied $^{9-18}$, topological bubbles do not contribute to current at the leading order.

We consider the regime of $e^{*} V \gg k_{B} T \gtrsim \hbar v_{\mathrm{p}} / L$, where the size $L_{V} \equiv \hbar v_{\mathrm{p}} /\left(e^{\star} V\right)$ of the dilutely injected anyons is much smaller than interferometer size $L$ and the injection of hole-like anyons at QPC1 is ignored; $v_{\mathrm{p}}$ is anyon velocity along the edges and $e^{\star} V$ should be much smaller than the FQH energy gap. Because of the dilute injection and $L_{V} \ll L$, anyon braiding is well defined in the interferometer. As shown below, the dependence of $I_{D_{3}}^{\text {int }}$ on $\Phi$ or on $V_{\mathrm{G}}$ provides a clear signature of the topological bubbles, consequently, $\theta^{*}=\pi v$ in both of the pure Aharonov-Bohm regime (where Coulomb interaction of the edge channels with bulk anyons localized inside the interferometer loop is negligible) and the Coulomb-dominated regime (where the interaction is strong $)^{22,25}$. Below, we first ignore bulk anyons.

Interference current. Employing the chiral Luttinger liquid theory ${ }^{26,27}$ for FQH edges and Keldysh Green's functions ${ }^{10,12}$, we compute $I_{D_{3}}^{\text {int }}\left(\propto \gamma_{1}^{2} \gamma_{2} \gamma_{3}\right)$ at the leading order in $\gamma$. There are four types of the processes mainly contributing to $I_{D_{3}}^{\text {int }} \simeq I_{D_{3}}^{I-1}+I_{D_{3}}^{\mathrm{I}-2}+I_{D_{3}}^{\mathrm{II}}+I_{D_{3}}^{\mathrm{III}}$; see Fig. 2b. For $e^{*} V \gg k_{\mathrm{B}} T \gg \hbar v_{\mathrm{p}} / L$, we obtain the analytical expression of $I_{D_{3}}^{\text {int: }}$ The interference current contributed by Type I-1 processes is 
a

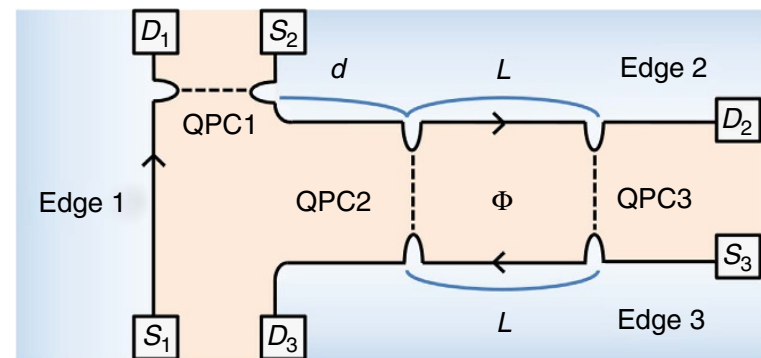

b Type I-1
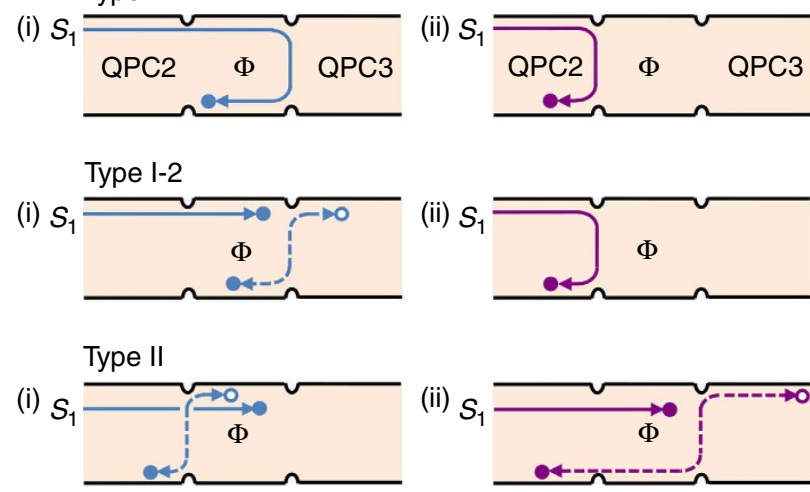

\section{Type III}

(i) $S_{1}$
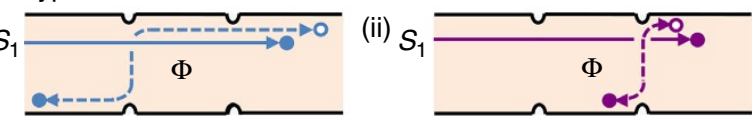

Figure 2 | Interferometry for detecting topological vacuum bubbles.

(a) In the setup, anyons move (see arrows) along $\mathrm{FQH}$ edge channel $i=1,2,3$ that connects source $S_{i}$ and drain $D_{i}$, and jump (dashed) between the channels via tunnelling at QPCs. The loop defined by Edges $i=2,3$, QPC2 and QPC3 encloses magnetic flux $\Phi$, forming a Fabry-Perot interferometer. Distance between QPC2 and QPC3 (QPC1) is $L(d)$. (b) Two interfering paths (i) and (ii) of each main interference process at $e^{*} V \gg k_{\mathrm{B}} T \gtrsim \hbar v_{\mathrm{p}} /$ L. Following Fig. 1, filled (empty) circles represent particle-like (hole-like) anyons and solid (dashed) lines denote propagation of an anyon injected from $S_{1}$ (anyon pair excitation at QPCs). Type II and III processes involve a topological vacuum bubble.

$I_{D_{3}}^{\mathrm{I}-1} \propto V^{v-2} T^{v-1} g(V, T) \cos \left(2 \pi \Phi / \Phi_{0}^{*}+e^{*} V L / \hbar v_{\mathrm{p}}\right)$, which by Type I-2 is $I_{D_{3}}^{\mathrm{I}-2} \propto V^{-1} g(V, T)\left(\sin ^{2} \pi v\right) \cos \left(2 \pi \Phi / \Phi_{0}^{*}\right)$, and those by Type II and III are

$$
\begin{gathered}
I_{D_{3}}^{\mathrm{II}} \simeq g(V, T) \frac{2 L+C(v) L_{T}}{\hbar v_{\mathrm{p}}}\left(\sin ^{2} \pi v\right) \cos \left(2 \pi \Phi / \Phi_{0}^{*}+\pi v\right), \\
I_{D_{3}}^{\mathrm{III}} \simeq g(V, T) \frac{C(v) L_{T}}{\hbar v_{\mathrm{p}}}\left(\sin ^{2} \pi v\right) \cos \left(2 \pi \Phi / \Phi_{0}^{*}-\pi v\right),
\end{gathered}
$$

where $g(V, T) \propto \gamma_{1}^{2} \gamma_{2} \gamma_{3}(V T)^{2 v-1} e^{-2 L / L_{T}}, e^{-2 L / L_{T}}$ is a thermal suppression factor, thermal length $L_{T} \equiv \hbar v_{\mathrm{p}} /\left(\pi v k_{\mathrm{B}} T\right)$ and $C(v=1 / 3) \simeq 0.43$; see Methods and the Supplementary Note 3 .

Type I-1 processes describe interference between two paths of an anyon moving from $S_{1}$ to $D_{3}$ via (i) QPC2 and (ii) QPC3, respectively. They were previously studied ${ }^{9}$.

In Type I-2, an anyon injected from $S_{1}$ interferes with a particle-like anyon excited at a QPC or annihilates a hole-like anyon; particle-like and hole-like anyons are pairwise excited thermally at QPCs. For example, consider the two following interfering histories: (i) an anyon is injected from $S_{1}$ to $D_{2}$, an anyon pair is excited at QPC3 and then the particle-like (hole-like) anyon of the pair moves to $D_{3}\left(D_{2}\right)$. (ii) An anyon is injected from $S_{1}$ to $D_{3}$ via QPC2 without any excitations. The hole-like anyon annihilates the injected anyon on Edge 2 in history (i) and the particle-like anyon of (i) interferes with the injected anyon of (ii) on Edge 3. The sum of such interference processes yields $I_{D_{3}}^{\mathrm{I}-2} \propto \sin ^{2} \pi v \cos \left(2 \pi \Phi / \Phi_{0}^{*}\right)$. The $\sin ^{2} \pi v$ factor appears, because relative locations of anyons on Edge 2 or 3 differ between the processes, leading to an exchange phase $\pm \pi v$, and because a process with an excitation (of a particle-like anyon moving to $D_{2}$ and a hole-like one to $D_{3}$ ) yields charge current in the opposite direction to another with its particle-hole conjugated excitation (of a particle to $D_{3}$ and a hole to $D_{2}$ ).

In Types II and III, a real anyon injected from $S_{1}$ moves to $D_{2}$ and a virtual anyon pair excited at QPC2 interferes with another at QPC3. The interference path effectively encloses the real anyon, forming a topological vacuum bubble (cf. Fig. 1). In Type II, when the real anyon is located on Edge 2 between QPC2 and QPC3, a virtual pair is excited at QPC2 in history (i) and at QPC3 in history (ii). Next, the hole-like (particle-like) anyon of each pair moves, for example, to $D_{2}\left(D_{3}\right)$. The interference of the two histories corresponds to the winding of a virtual anyon around the real one and $\Phi$, forming a topological bubble with interference phase $\pm\left(2 \pi \Phi / \Phi_{0}^{*}+2 \pi v\right)$; \pm depends on whether the hole-like anyon moves to $D_{2}$ or $D_{3}$. In the interference, the winding of a virtual anyon around the real one effectively occurs through the exchanges of the positions of the anyons in each of Edges 2 and 3, as relative locations of anyons on Edges 2 and 3 differ between (i) and (ii) (see Supplementary Fig. 2 and Supplementary Note 6). This interference is accompanied by a partner process. The latter has a bubble that winds around $\Phi$ (gaining phase $\pm 2 \pi \Phi / \Phi_{0}^{*}$ ), but not around a real anyon. The two partner processes partially cancel each other, yielding $I_{D_{3}}^{\mathrm{II}} \propto \sin \pi v \cos \left(2 \pi \Phi / \Phi_{0}^{*}+\pi v\right)$; the remaining $\sin \pi v$ factor in equations (3) and (4) has a similar origin to the $\sin ^{2} \pi v$ factor of $I_{D_{3}}^{\mathrm{I}-2}$.

In Type III, the two interfering histories are as follows: (i) a virtual pair is excited at QPC2 before a real anyon injected from $S_{1}$ arrives at QPC2 and (ii) another pair is excited at QPC3 after the real one arrives at QPC3. The ensuing chronological sequence on Edge 2 is opposite to Type II: an anyon excited at QPC2 arrives at QPC3; the real one arrives at QPC3; a pair is excited at QPC3. The resulting topological bubble effectively winds around the real anyon in the direction opposite to its winding around $\Phi$, yielding a phase $\pm\left(2 \pi \Phi / \Phi_{0}^{*}-2 \pi v\right)$. Partial cancellation of the bubble and its partner leads to $I_{D_{3}}^{\mathrm{III}} \propto \sin \pi v \cos \left(2 \pi \Phi / \Phi_{0}^{*}-\pi v\right)$. The factor $2 L+C L_{T}$ of $I_{D_{3}}^{\mathrm{II}}\left(C \dot{L}_{T}\right.$ in $\left.I_{D_{3}}^{\mathrm{III}}\right)$ in equation (3) (equation (4)) comes from the time window compatible with the chronological sequence on Edge 2.

Type II and III processes of topological bubbles do not affect any observables at $v=1$ (fermions), due to full cancellation between partner bubbles (the linked cluster theorem). They are distinct from I-2. I-2 processes produce, for example, non-vanishing current noise $\left\langle\left(I_{D_{3}}^{\text {int }}-\left\langle I_{D_{3}}^{\text {int }}\right\rangle\right)^{2}\right\rangle$ at $v=1$, as the particle-hole conjugated excitations (mentioned before) equally contribute to the noise (although the contributions of the conjugations to $I_{D_{3}}^{\mathrm{I}-2}$ cancel each other, leading to $I_{D_{3}}^{\mathrm{I}-2}=0$ ).

In the more general regime of $e^{*} V \gg k_{\mathrm{B}} T \gtrsim \hbar v_{\mathrm{p}} / L$, we employ the parametrization

$$
I_{D_{3}}^{\text {int }} \propto \cos \left(2 \pi \Phi / \Phi_{0}^{*}+\theta\right) .
$$

The phase $\theta$ is determined by competition between the various contributions to $I_{D_{3}}^{\text {int }}$ and contains information about statistics phase $\pi v$. At $e^{*} V \gg k_{\mathrm{B}} T \gtrsim \hbar v_{\mathrm{p}} / L, I_{D_{3}}^{\mathrm{II}}+I_{D_{3}}^{\mathrm{III}}$ is much larger than $I_{D_{3}}^{\mathrm{I}-1}+I_{D_{3}}^{\mathrm{I}-2}$ and dominates $I_{D_{3}}^{\mathrm{int}}$, because the interfering anyon of Types I-1 and I-2 is voltage biased and has width $L_{V} \propto V^{-1}$ much narrower than the thermal anyon excitations (whose width $L_{T} \propto T^{-1}$ ) of II and III, showing much weaker interference. From 
$I_{D_{3}}^{\mathrm{II}}$ and $I_{D_{3}}^{\mathrm{III}}$, we find

$$
\begin{aligned}
\theta & \simeq \arctan \left[\frac{L}{L+C(v) L_{T}} \tan \left(\pi v+(1-2 v) \arctan \frac{2 L_{V}}{L_{T}}\right)\right] \\
& \rightarrow \arctan \left[\frac{L}{L+C(v) L_{T}} \tan \pi v\right] \quad \text { as } T / V \rightarrow 0 \\
& \rightarrow \pi v \quad \text { as } L_{T} / L \rightarrow 0 \text { and } T / V \rightarrow 0 .
\end{aligned}
$$

The $\arctan 2 L_{V} / L_{T}$ term represents an error in the braiding phase $2 \pi v$ of the topological bubbles. It occurs when the size $L_{V}$ of a real anyon is not sufficiently smaller than the winding radius of a virtual anyon around the real one. It is negligible at $e^{*} V \gg k_{\mathrm{B}} T$, as the radius is effectively large when $L_{V} \ll L_{T}$; those corresponding to the error are ignored in equations (3) and (4), and are shown in Supplementary Note 3. For $e^{*} V \gg k_{\mathrm{B}} T \gg \hbar v_{\mathrm{p}} / L, I_{D_{3}}^{\mathrm{II}}$ dominates $I_{D_{3}}^{\mathrm{int}}$ and $\theta \rightarrow \pi v$. Remarkably, $\theta$ depends on $T$, contrary to common practice in electron interferometry ${ }^{28}$.

Coulomb-dominated regime. In experimental situations of a Fabry-Perot interferometer in the FQH regime, it is expected that there exist bulk anyons localized inside the interferometer loop. There are two regimes of Fabry-Perot interferometers, the pure Aharonov-Bohm regime and the Coulomb-dominated regime. In the former regime, Coulomb interaction between the bulk anyons and the edge of the interferometer is negligible, whereas it is crucial in the latter ${ }^{25}$. The Fabry-Perot interferometers of recent experiments ${ }^{17,20-23}$ in the $\mathrm{FQH}$ regime are in the Coulombdominated regime. Below we compute the interference current $I_{D_{3}}$ in the presence of the Coulomb interaction and show that equation (6) is applicable to both of the pure Aharonov-Bohm regime and the Coulomb-dominated regime.

For $e^{*} V \gg k_{\mathrm{B}} T \gtrsim \hbar v_{\mathrm{p}} / L$, we numerically compute $I_{D_{3}}^{\text {int }}\left(\propto \gamma_{1}^{2} \gamma_{2} \gamma_{3}\right)$ in Fig. 3, combining our theory with the capacitive interaction model ${ }^{25}$ that successfully describes thermally fluctuating bulk anyons and the interaction (see the Method and Supplementary Note 5). We find the gate-voltage dependence of $I_{D_{3}}^{\text {int }} \propto \cos \left(2 \pi V_{\mathrm{G}} / V_{\mathrm{G}, 0}+\theta\right)$ with periodicity $V_{\mathrm{G}, 0}$ in the Coulomb-dominated regime and $I_{D_{3}}^{\text {int }} \propto \cos \left(2 \pi \Phi / \Phi_{0}+\theta\right)$ in the pure Aharonov-Bohm limit; here the periodicity of the $\Phi$ dependence is $\Phi_{0} \equiv h / e$ rather the period $\Phi_{0}^{*}$ of equation (5), because of the fluctuation of the number of bulk anyons ${ }^{9,19}$. In both the regimes, the interference processes discussed before (Fig. 2) appear in the same manner; hence, $\theta$ satisfies the analytic expression in equation (6) (cf. Fig. 3c).

How to measure the phase $\theta$. Experimental measurements of $\theta$ can be affected by possible side effects, including the externalparameter (magnetic fields, gate voltages and bias voltages) dependence of the size, shape, QPC tunnelling and bulk anyon excitations. Below we propose how to detect $\theta$ with avoiding the side effects, using the setup in Fig. 2a.

The phase $\theta$ is experimentally measurable, by comparing $I_{D_{3}}^{\text {int }}$ with a reference current $I_{\text {Ref, }}^{\text {int }} D_{3} . I_{\text {Ref, }}^{\text {int }} D_{3}$ is measured at $D_{3}$ in the same setup under the same external parameters (temperature, gate voltages, magnetic field and so on) with $I_{D_{3}}^{\text {int }}$, but with applying infinitesimal bias voltage $V_{\text {ref }} / 2$ to $S_{2}$ and $-V_{\text {ref }} / 2$ to $S_{3}$ and keeping $S_{1}$ and all $D_{i}$ 's grounded ${ }^{9}$ ( $c f$. Supplementary Note 4). In any regimes $I_{D_{3}}^{\text {int }}$ shows the same interference pattern with $I_{\text {Ref }}^{\text {int }} D_{3}$, but is phase-shifted from $I_{\text {Ref, }}^{\text {int }} D_{3}$ by $\theta$; $I_{\text {Ref, } D_{3}}^{\text {int }} \propto \cos 2 \pi V_{\mathrm{G}} / V_{\mathrm{G}, 0}\left(I_{\text {Ref }, D_{3}}^{\text {int }} \propto \cos 2 \pi \Phi / \Phi_{0}\right)$ in the Coulombdominated (pure Aharonov-Bohm) limit. Importantly, the side effects modify $I_{D_{3}}^{\text {int }}$ and $I_{\mathrm{Ref}, D_{3}}^{\text {int }}$ in the same manner; hence, the phase shift between the patterns remains as $\theta$. a
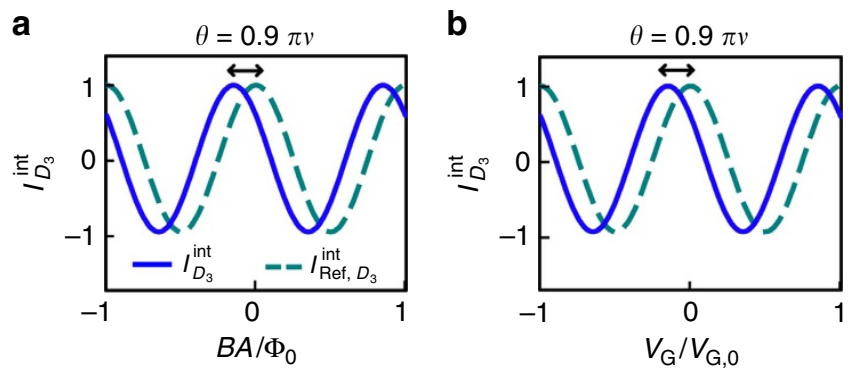

C

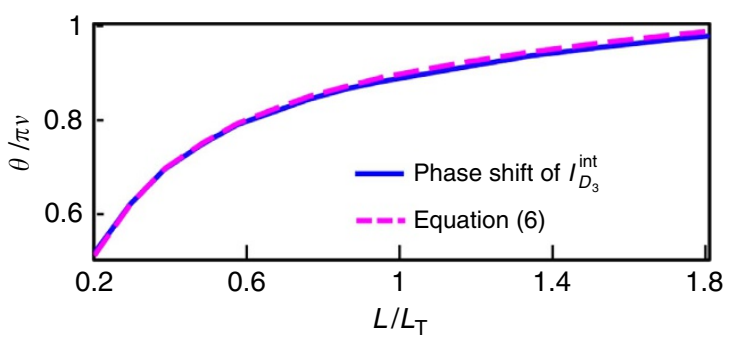

Figure 3 | Detection of anyon phase $\boldsymbol{\pi} \boldsymbol{\nu}$ from interference phase shift $\boldsymbol{\theta}$. (a) Dependence of $I_{D_{3}}^{\text {int }}$ (blue, normalized) and $I_{\text {Ref, } D_{3}}^{\text {int }}$ (cyan, normalized) on $\Phi$ in the pure Aharonov-Bohm regime and (b) their dependence on $V_{G}$ in the Coulomb dominated regime. We choose $v=1 / 3, T=30 \mathrm{mK}$, $e^{\star} V=45 \mu \mathrm{eV}, L=3 \mu \mathrm{m}$ and $v_{\mathrm{p}}=10^{4} \mathrm{~m} \mathrm{~s}^{-1}\left(L / L_{T}=1.2\right.$ and $\left.L / L_{V}=20\right)$; see Supplementary Note 5 for the Coulomb interaction parameter of the regimes. For these parameters, the phase shift $\theta$ between $l_{D_{3}}^{\text {int }}$ and $l_{\mathrm{Ref}, D_{3}}^{\text {int }}$ is $0.9 \pi v$. (c) Dependence of $\theta$ on $T$. The same parameters (except $T$ ) with (a) and (b) are chosen. $\theta \rightarrow \pi v$ as $T$ increases (yet $e^{*} V \gg k_{B} T \gg \hbar v_{p} / L$ ). In both the pure Aharonov-Bohm regime and the Coulomb-dominated regime, the same numerical result (blue curve) of $\theta(T)$, which agrees with equation (6) (magenta), is obtained.

The fractional statistics phase is directly and unambiguously identifiable in experiments, by observing $\theta \rightarrow \pi v$ at $e^{*} V \gg$ $k_{\mathrm{B}} T \gg \hbar v_{\mathrm{p}} / L$ with excluding the side effects as above, or one applies the fit function of $\arctan \left[A_{1} /\left(1+A_{2} / T\right)\right]$ with fit parameters $A_{1}$ and $A_{2}$ to measured data of $\theta(T)$ and extracts $\arctan A_{1}=\pi v$ from the fit ( $c f$. the second line of equation (6)). Observation of $\theta=\pi v$ or $\theta(T)$ will suggest a strong evidence of anyon braiding and topological bubbles.

The parameters in Fig. 3 are experimentally accessible ${ }^{17,20-23}$. For the QPCs, there are constraints (i) that the number of voltagebiased anyons injected through QPC1 is at most one in the interferometer loop at any instance (to ensure that the braiding phase of a topological vacuum bubble is $2 \pi v$ ), (ii) that the anyon tunnelling probabilities at QPC2 and QPC3 are sufficiently small (to ensure that the double winding of an anyon along the interferometer loop is negligible) and (iii) that anyon tunnelling (rather than electron tunnelling) occurs at the QPCs. The constraint (i) is satisfied when the anyon tunnelling probability at QPC1 is $<\hbar v_{\mathrm{p}} /\left(2 L e^{\star} V\right)$, which is $\sim 0.05$ under the parameters. To achieve the constraints (ii) and (iii), each tunnelling probability of QPC2 and QPC3 is typically set to be 0.4 in experiments ${ }^{22,29}$; then the amplitude of the double winding is smaller than that of the single winding by the factor $0.4 \exp \left(-2 L / L_{T}\right)$, which is $\sim 0.04$ at $30 \mathrm{mK}$. With the constraints we estimate the amplitude of $I_{D_{3}}^{\text {int }} \lesssim\left(v e^{2} V / h\right)\left(\hbar v_{\mathrm{p}} /\left(2 L e^{*} V\right)\right) 0.4 \exp \left(-2 L / L_{T}\right)$, which is $1.5 \mathrm{pA}$ at $30 \mathrm{mK}$ and $0.6 \mathrm{pA}$ at $40 \mathrm{mK}$ under the parameters. It is noteworthy that $\theta=0.9 \pi v$ is reached at $30 \mathrm{mK}$, while $\theta=0.95 \pi v$ at $40 \mathrm{mK}$ under the parameters. The estimation is within a measurable range in experiments, where current $\gtrsim 0.5 \mathrm{pA}$ is well detectable ${ }^{30}$. 
We remark that the above strategy of detecting the fractional statistics phase is equally applicable to the more general quantum Hall regime ${ }^{22}$ of filling factor $v^{\prime}=v+v_{0}$, in which the edge channels from the integer filling $v_{0}$ are fully transmitted through the QPCs, while the channel from the fractional filling $v$ forms the interferometry in Fig. 2. For this case we compute $I_{D_{3}}^{\text {int }}$ and $I_{\text {Ref, }}^{\text {int }} D_{3}$, and find that in both of the pure Aharonov-Bohm regime and the Coulomb-dominated regime the interference-pattern phase shift between them is identical to the phase $\theta$ of the $v_{0}=0$ case discussed in equation (6) and Fig. 3 (cf. Supplementary Note 5).

Certain anyonic vacuum bubbles involve topological braiding and affect physical observables surprisingly, contrary to vacuum bubbles of bosons and fermions. They can be detected with current experiment tools, which will provide an unambiguous evidence of anyonic fractional statistics. We expect that they are relevant also for other filling fractions $v=p /(2 n p+1)$, non-Abelian anyons ${ }^{17,21}$ and topological quantum computation setups $^{31}$.

\section{Methods}

Hamiltonian for the interferometer. We present the Hamiltonian for the setup. We recall the chiral Luttinger liquid theory for $\mathrm{FQH}$ edges.

The Hamiltonian $H=\sum_{i} H_{\text {edge }, i}+H_{\text {tun }}$ for the interferometer in Fig. 2a consists of $H_{\mathrm{edge}, i}$ for edge channel $i$ and $H_{\text {tun }}$ for anyon tunnelling at QPCs. Edge channel 1 is biased by $V$ and its Hamiltonian, employing the bosonization ${ }^{26}$ for chiral Luttinger liquids, is given by

$$
H_{\text {edge }, 1}=\frac{\hbar v_{\mathrm{p}}}{4 \pi v} \int_{-\infty}^{\infty} d x:\left(\partial_{x} \phi_{1}(x)\right)^{2}:+e^{*} V \hat{N}_{1} .
$$

For the other unbiased channels, $H_{\text {edge, } i=2,3}=\left(\hbar v_{\mathrm{p}} / 4 \pi v\right) \int_{-\infty}^{\infty} d x:\left(\partial_{x} \phi_{i}(x)\right)^{2}$ : Here, $e^{*}>0, \hat{N}_{i}$ is the anyon number operator of channel $i$ and $\phi_{i}(x)$ is the bosonic field of channel $i$ at position $x$, which describes the plasmonic excitation of anyons. The tunnelling Hamiltonian is $H_{\text {tun }}=T_{1}+T_{2}^{\downarrow}+T_{3}^{\downarrow}+$ h.c., $T_{1}^{\rightarrow}$ is the operator from Edge channel 1 to 2 at QPC 1, $T_{2}^{\downarrow}$ from Edge 2 to 3 at QPC2 and $T_{3}^{\downarrow}$ from Edge 2 to 3 at QPC3. These are written as

$$
\begin{gathered}
T_{1}^{\rightarrow}(t)=\gamma_{1} \Psi_{2}^{\dagger}(0, t) \Psi_{1}(0, t), \\
T_{2}^{\downarrow}(t)=\gamma_{2} e^{-i \pi \Phi / \Phi_{0}^{*}} \Psi_{3}^{\dagger}(L, t) \Psi_{2}(d, t), \\
T_{3}^{\downarrow}(t)=\gamma_{3} e^{i \pi \Phi / \Phi_{0}^{*}} \Psi_{3}^{\dagger}(0, t) \Psi_{2}(d+L, t),
\end{gathered}
$$

where $\Psi_{i}^{\dagger}(x, t)=F_{i}^{\dagger}(t) e^{-i \phi_{i}(x, t)} / \sqrt{2 \pi a}$ creates an (particle-like) anyon at position $x$ and time $t$ on Edge $i, a$ is the short-length cutoff, $\gamma_{i}$ is the tunnelling strength at QPC $i$ (chosen as real) and the Aharonov-Bohm flux $\Phi$ enclosed by Edges $i=2,3$, QPC2 and QPC3 is attached to $T_{2}^{\downarrow}$ and $T_{3}^{\downarrow}$, respectively, under certain gauge transformation; the dynamical phase common to the all edge channels is absorbed to $2 \pi \Phi / \Phi_{0}^{*}$. The Klein factor $F_{i}^{\dagger}$ increases the number of anyons on Edge $i$ by 1 and satisfies $F_{i}^{\dagger} F_{i}=F_{i} F_{i}^{\dagger}=1,\left[N_{i} F_{j}^{\dagger}\right]=\delta_{i j} F_{i}^{\dagger}, F_{1}(t)=F_{1}(0) e^{-i e^{*} V t / \hbar}$ and $\left[\phi_{i}, F_{j}\right]=0$.

The exchange rule in equation (1) is described by $\phi_{i}$ and $F_{i}$. On Edge $i$, it is satisfied by

$$
\left[\phi_{i}\left(x_{1}\right), \phi_{j}\left(x_{2}\right)\right]=i \pi v \delta_{i j} \operatorname{sgn}\left(x_{1}-x_{2}\right) \text {. }
$$

The exchange rule between anyons on different edges is achieved with the commutators of $F_{i}$,

$$
F_{i} F_{j}=F_{j} F_{i} e^{-i \pi v \operatorname{sgn}(i-j)}, \quad F_{i}^{\dagger} F_{j}=F_{j} F_{i}^{\dagger} e^{i \pi v \operatorname{sgn}(i-j)} .
$$

A conventional way ${ }^{32}$ for obtaining the commutators is to think of an extended edge connecting the different channel segments with no twist (cf. Fig. 4). The connection should preserve the chiral propagation direction of the channels. The exchange rule $\Psi\left(x_{1}\right) \Psi\left(x_{2}\right)=\Psi\left(x_{2}\right) \Psi\left(x_{1}\right) e^{-i \pi v s g n\left(x_{1}-x_{2}\right)}$ of anyons of the extended edge agrees with equations (11) and (12).

We consider the regime of weak tunnelling of anyons and treat $H_{\text {tun }}$ as a perturbation on $\sum_{i} H_{\text {edge, } i}$. Perturbation theory is applicable ${ }^{24}$ in the renormalization group sense, when $e^{\star} V$ and $k_{\mathrm{B}} T$ are higher than $C \gamma^{1 /(1-v)}, C$ being a non-universal constant.

The current $I_{D_{3}}$ is expressed as $I_{D_{3}}=i e^{*}\left[N_{3}, H\right]=i e^{*}\left(T_{2}^{\downarrow}-T_{2}^{\uparrow}+T_{3}^{\downarrow}-T_{3}^{\uparrow}\right) . I_{D_{3}}$ is decomposed, $I_{D_{3}}=I_{D_{3}}^{\text {dir }}+I_{D_{3}}^{\text {int }}$, into direct current $I_{D_{3}}^{\text {dir }} \propto \gamma_{1}^{2} \gamma_{2}^{2}, \gamma_{1}^{2} \gamma_{3}^{2}$ and interference current $I_{D_{3}}^{\text {int }} \propto \gamma_{1}^{2} \gamma_{2} \gamma_{3}$ depending on $\Phi$ (the leading-order contribution). Equations (3) and (4) are obtained by employing Keldysh Green's function technique with semiclassical approximation (see Supplementary Fig. 1 and Supplementary Notes 1,2 and 3).

Coulomb interaction. In the presence of Coulomb interaction between bulk anyons and edge channels, we compute $I_{D_{3}}^{\text {int }}$, combining our chiral Luttinger liquid

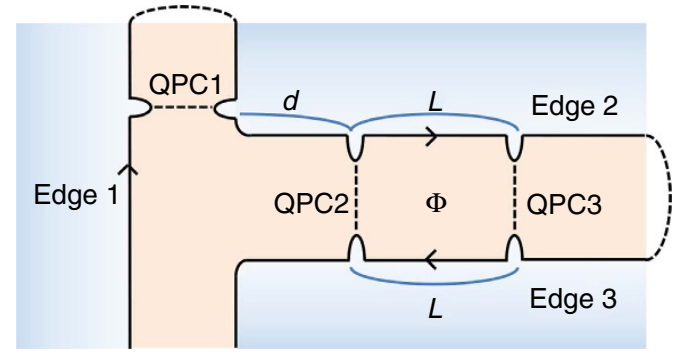

Figure 4 | Extended edge channel scheme. It is obtained by connecting the edge channels of the setup in Fig. 2a. The connection is represented by dashed arcs, whereas anyon propagation direction and anyon tunnelling at QPCs are represented by arrows and dashed lines, respectively.

theory with the capacitive interaction model ${ }^{25}$ that successfully describes the Coulomb-dominated regime. The interferometer Hamiltonian $H=\sum_{i} H_{\text {edge }, i}+H_{\text {tun }}$ is modified by the Coulomb interaction as

$$
\begin{aligned}
H & \rightarrow H+U_{\text {bulk }} Q_{\text {bulk }}^{2} \\
& +U_{\text {int }} Q_{\text {bulk }} \int_{0}^{L} d x\left(: \partial_{x} \phi_{2}(x+d):+: \partial_{x} \phi_{3}(x):\right) \\
& =\sum_{i=1,2,3} \frac{\hbar v_{\mathrm{p}}}{4 \pi v} \int_{-\infty}^{\infty} d x:\left(\partial_{x} \bar{\phi}_{i}(x)\right)^{2}:+H_{\text {bulk }}+H_{\text {tun }} .
\end{aligned}
$$

Here, $Q_{\text {bulk }}=v B A_{\text {area }} / \Phi_{0}+v N_{\mathrm{L}}-\bar{q}$ is the number of the net charges localized within the interferometer bulk (inside the interference loop), $A_{\text {area }}$ is the area of the interferometer, $N_{\mathrm{L}}$ is the net number of quasiparticles minus quasiholes and $\bar{q}$ is the number of positive background charges induced by the gate voltage applied to the interferometer. $U_{\text {int }}$ is the strength of Coulomb interaction between the charges of the interferometer edge and the charges localized in the interferometer bulk, and $U_{\text {bulk }}$ is the strength of interaction between the bulk charges. In the second equality of equation (13), we introduce a boson field $\bar{\phi}_{i}$ for each Edge $i$, $\bar{\phi}_{i}(x)=\phi_{i}(x)+\frac{2 \pi v}{\hbar v_{\mathrm{p}}} U_{\text {int }} Q_{\text {bulk }} \int_{-\infty}^{x} K_{i}\left(x^{\prime}\right) d x^{\prime}$, where $K_{2}(x)=1$ for $d<x<d+L$, $K_{3}(x)=1$ for $0<x<L$ and $K_{i}(x)=0$ otherwise. The second term of $\bar{\phi}$ describes the charges $-\frac{2 \pi v}{\hbar v_{\mathrm{p}}} U_{\text {int }} Q_{\text {bulk }}$ induced per unit length by the interaction. In equation (13), the Hamiltonian is quadratic in $\bar{\phi}_{i}$ and has $H_{\text {bulk }}=\left(U_{\text {bulk }}-\frac{2 \pi v L}{\hbar v_{p}} U_{\text {int }}^{2}\right) Q_{\text {bulk }}^{2}$. It is noteworthy that $U_{\text {bulk }}-\frac{2 \pi v L}{\hbar v_{\mathrm{p}}} U_{\text {int }}^{2}>0$. The main interference signal $I_{D_{3}}$ and the reference signal $I_{\mathrm{Ref},}^{\mathrm{int}} \mathrm{D}_{3}$ are computed by taking ensemble average over the thermal fluctuations of $N_{\mathrm{L}}$ (see Supplementary Note 5).

\section{References}

1. Fetter, A. L. \& Walecka, J. D. Quantum Theory Of Many-Particle Systems (McGraw-Hill, 1971)

2. Leinaas, J. M. \& Myrheim, J. On the theory of identical particles. Il Nuovo Cimento B Ser 37, 1-23 (1977).

3. Arovas, D., Schrieffer, J. R. \& Wilczek, F. Fractional statistics and the quantum Hall effect. Phys. Rev. Lett. 53, 722-723 (1984).

4. Stern, A. Anyons and the quantum Hall effect-a pedagogical review. Ann. Phys. 1, 204-249 (2008).

5. Goldman, V. J. \& Su, B. Resonant tunneling in the quantum Hall regime: measurement of fractional charge. Science 267, 1010-1012 (1995).

6. De-Picciotto, R. et al. Direct observation of a fractional charge. Nature 389, 162-164 (1997).

7. Saminadayar, L., Glattli, D. C., Jin, Y. \& Etienne, B. Observation of the e/3 fractionally charged Laughlin quasiparticle. Phys. Rev. Lett. 79, 2526-2529 (1997).

8. Dolev, M., Heiblum, M., Umansky, V., Stern, A. \& Mahalu, D. Observation of a quarter of an electron charge at the $v=5 / 2$ quantum Hall state. Nature 452, 829-834 (2008).

9. Chamon, C. D. C., Freed, D. E., Kivelson, S. A., Sondhi, S. L. \& Wen, X. G. Two point-contact interferometer for quantum Hall systems. Phys. Rev. B 55, 2331-2343 (1997).

10. Safi, I., Devillard, P. \& Martin, T. Partition noise and statistics in the fractional quantum Hall effect. Phys. Rev. Lett. 86, 4628-4631 (2001).

11. Vishveshwara, S. Revisiting the Hanbury Brown-Twiss setup for fractional statistics. Phys. Rev. Lett. 91, 196803 (2003).

12. Kim, E.-A., Lawler, M., Vishveshwara, S. \& Fradkin, E. Measuring fractional charge and statistics in fractional quantum Hall fluids through noise experiments. Phys. Rev. B 74, 155324 (2006).

13. Law, K. T., Feldman, D. E. \& Gefen, Y. Electronic Mach-Zehnder interferometer as a tool to probe fractional statistic. Phys. Rev. B 74, 045319 (2006).

14. Feldman, D. E., Gefen, Y., Kitaev, A., Law, K. T. \& Stern, A. Shot noise in an anyonic Mach-Zehnder interferometer. Phys. Rev. B 76, 085333 (2007). 
15. Campagnano, G. et al. Hanbury Brown-Twiss interference of anyons. Phys. Rev. Lett. 109, 106802 (2012).

16. Kane, C. L. Telegraph noise and fractional statistics in the quantum Hall effect. Phys. Rev. Lett. 90, 226802 (2003).

17. An, S. et al. Braiding of abelian and non-abelian anyons in the fractional quantum Hall effect. Preprint at http://arXiv.org/abs/1112.3400 (2011).

18. Rosenow, B. \& Simon, S. H. Telegraph noise and the Fabry-Perot quantum Hall interferometer. Phys. Rev. B 85, 201302 (2012).

19. Kivelson, S. Semiclassical theory of localized many-anyon states. Phys. Rev. Lett. 65, 3369-3372 (1990).

20. Camino, F. E., Zhou, W. \& Goldman, V. J. e/3 Laughlin quasiparticle primaryfilling =1/3 interferometer. Phys. Rev. Lett. 98, 076805 (2007).

21. Willett, R. L., Pfeiffer, L. N. \& West, K. W. Measurement of filling factor 5/2 quasiparticle interference with observation of charge e/4 and e/2 period oscillations. Proc. Natl Acad. Sci. USA 106, 8853-8858 (2009).

22. Ofek, N. et al. Role of interactions in an electronic Fabry Perot interferometer operating in the quantum Hall effect regime. Proc. Natl Acad. Sci. USA 107, 5276-5281 (2010).

23. McClure, D. T., Chang, W., Marcus, C. M., Pfeiffer, L. N. \& West, K. W. FabryPerot interferometry with fractional charges. Phys. Rev. Lett. 108, 256804 (2012).

24. Kane, C. L. \& Fisher, M. P. A. Transmission through barriers and resonant tunneling in an interacting one-dimensional electron gas. Phys. Rev. B 46, 15233-15262 (1992)

25. Halperin, B., Stern, A., Neder, I. \& Rosenow, B. Theory of the Fabry-Perot quantum Hall interferometer. Phys. Rev. B 83, 155440 (2011).

26. von Delft, J. \& Schoeller, H. Bosonization for beginners---refermionization for experts. Ann. Phys. (Leipzig) 7, 225-306 (1998).

27. Wen, X. G. Chiral Luttinger liquid and the edge excitations in the fractional quantum Hall states. Phys. Rev. B 41, 12838-12844 (1990).

28. Ji, Y. et al. An electronic Mach Zehnder interferometer. Nature 422, 415-418 (2003).

29. Griffiths, T. G. et al. Evolution of quasiparticle charge in the fractional quantum Hall regime. Phys. Rev. Lett. 85, 3918-3921 (2000).

30. Comforti, E. et al. Bunching of fractionally charged quasiparticles tunnelling through high-potential barriers. Nature 416, 515-518 (2002).

31. Nayak, C., Simon, S. H., Stern, A., Freedman, M. \& Sarma, S. D. Non-Abelian anyons and topological quantum computation. Rev. Mod. Phys. 80, 1083-1159 (2008).
32. Guyon, R., Devillard, P., Martin, T. \& Safi, I. Klein factors in multiple fractional quantum Hall edge tunneling. Phys. Rev. B 65, 153304 (2002).

\section{Acknowledgements}

H.-S.S. thanks Eddy Ardonne, Hyungkook Choi, Yunchul Chung, Dmitri Feldman, Woowon Kang, Kirill Shtengel and Joost Slingerland for useful discussion. We thank the support by Korea NRF (grant numbers NRF-2010-00491 and NRF-2013R1A2A2A01007327 to H.-S.S.), by KAIST-HRHRP (C.H.) and by DFG (grant number RO 2247/8-1 to Y.G.).

\section{Author contributions}

C.H. has conceived the concept of topological vacuum bubbles, performed the detailed calculation, analysed the Coulomb-dominated regime and wrote the paper. J.P. has performed the analysis and wrote the paper. Y.G. has conceived the interferometry setup and wrote the paper. H.-S.S. has conceived the concept of topological vacuum bubbles and the interferometry setup, analysed the Coulomb-dominated regime, wrote the paper and supervised the project.

\section{Additional information}

Supplementary Information accompanies this paper at http://www.nature.com/ naturecommunications

Competing financial interests: The authors declare no competing financial interests.

Reprints and permission information is available online at http://npg.nature.com/ reprintsandpermissions/

How to cite this article: Han, C. et al. Topological vacuum bubbles by anyon braiding. Nat. Commun. 7:11131 doi: 10.1038/ncomms11131 (2016)

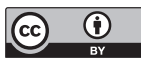

This work is licensed under a Creative Commons Attribution 4.0 International License. The images or other third party material in this article are included in the article's Creative Commons license, unless indicated otherwise in the credit line; if the material is not included under the Creative Commons license, users will need to obtain permission from the license holder to reproduce the material. To view a copy of this license, visit http://creativecommons.org/licenses/by/4.0/ 